\title{
Enfrentamento Religioso-Espiritual de Mães com Bebê em Unidade de Terapia Intensiva Neonatal
}

\author{
Gisele Fernandes de Lima Foch ${ }^{1}$ \\ Andressa Melina Becker da Silva \\ Sônia Regina Fiorim Enumo \\ Programa de Pós-Graduação em Psicologia da Pontifícia Universidade Católica \\ de Campinas, Campinas, SP, Brasil
}

\begin{abstract}
Resumo
A Teoria Motivacional do Coping (TMC) define o enfrentamento como a forma com que as pessoas regulam o comportamento, a emoção e a orientação motivacional frente ao estressor. A internação do filho em Unidade de Terapia Intensiva Neonatal (UTIN) é uma condição estressante, sendo frequente o uso do coping religioso-espiritual. Analisou-se o processo de enfrentamento de 20 mães (17-39 anos), com bebês internados em UTIN ( $M=10$ dias). Foi aplicada a versão brasileira da RCOPE Scale (Religious Coping Questionnaire), denominada Escala de Coping Religioso-Espiritual - CRE (87 itens), organizados em 8 fatores de CREP (positivo) e 4 de CREN (negativo). Analisaram-se suas relações com categorias de enfrentamento adaptativas (Autoconfiança, Busca de suporte, Resolução de problemas, Busca de Informações, Acomodação, Negociação) e mal adaptativas (Delegação, Isolamento, Desamparo, Fuga, Submissão e Oposição). Os índices da Escala CRE ficaram na média, com predomínio de CREN e maior frequência de Posicionamento Negativo frente a Deus, e de estratégias mal adaptativas, como Submissão, Fuga e Oposição. Houve correlações entre CREP e famílias adaptativas; e entre CREN e famílias mal adaptativas. Foi possível identificar o uso do coping religioso-espiritual nesta amostra e analisar suas relações com o processo adaptativo, ampliando as possibilidades de análise da Escala CRE.
\end{abstract}

Palavras-chave: Coping, estresse, Unidades de Terapia Intensiva Neonatal, Escala de Coping Religioso-Espiritual.

Endereço para correspondência: Avenida Goffredo Teixeira da Silva Telles, 763, Araras, SP, Brasil 13607350. Fone: (019) 99154-7598/(019) 3321-9777. E-mail: gi_flima@yahoo.com.br, andressa_becker@hotmail. com e sonia.enumo@puc-campinas.edu.br.

Trabalho derivado da dissertação de mestrado da primeira autora, orientada pela terceira: Foch, G. F. L. (2015). Enfrentamento religioso-espiritual de mães de bebês em Unidade de Terapia Intensiva Neonatal. Pontifícia Universidade Católica de Campinas, Campinas, SP."

Agradecimentos: À Coordenação de Aperfeiçoamento de Pessoal de Nível Superior - CAPES (bolsa de mestrado para primeira autora; bolsa de doutorado para segunda autora); Conselho Nacional de Desenvolvimento Científico e Tecnológico - CNPq (bolsa de produtividade em pesquisa para terceira autora); aos estudantes de Psicologia - Carla C. Martins, Jéssica S. Câmara e Rebeca de Menezes pelo apoio na coleta de dados; à Direção do hospital; às mães que participaram da pesquisa. 


\title{
Mothers' Spiritual-Religious Coping with Baby in Neonatal Intensive Care Unit
}

\begin{abstract}
The Motivational Theory of Coping (MTC) defines coping as how people regulate behavior, emotion and motivational orientation against the stressor. The child's hospitalization in the Neonatal Intensive Care Units (NICU) is a stressful condition, with frequent use of spiritual and religious coping. It was analyzed the 20 mothers (17-39 years) coping process with babies admitted to NICU ( $M=10$ days). The Brazilian version of RCOPE, the Spiritual/Religious Coping Scale (SRCOPE) was applied (87 items), organized into 8 factors of SRCOPE-P (positive) and 4 SRCOPE-N (negative). Their relations with adaptive coping categories (Self-reliance, Support Seeking, Problem Solving, Information Seeking, Accommodation, Negotiation) and maladaptive (Delegation, Isolation, Helplessness, Escape, Submission and Opposition) were analyzed. The indices of SRCOPE were on average, with a predominance of SRCOPE-N and higher frequency of Negative Positioning front of God, and prevalence of maladaptive strategies such as Submission, Escape and Opposition. There were correlations between SRCOPE-P and adaptive families; and between SRCOPE-N and maladaptive families. It was possible identify the use of spiritual and religious coping in this sample, and analyze its relations with adaptive process, expanding the possibilities of analysis of SRCOPE Scale.
\end{abstract}

Keywords: Coping, stress, Neonatal Intensive Care Units, Spiritual-Religious Coping Scale.

\section{Afrontamiento Religioso y Espiritual de las Madres con Bebé en la Unidad de Cuidados Intensivos Neonatales}

\section{Resumen}

Teoría Motivacional de Afrontamiento (TMC) define afrontamiento cómo forma de regular comportamiento, emociones y orientación motivacional frente al factor estresante. La hospitalización de un hijo en la unidad de cuidados intensivos neonatales (UCIN) es una condición estresante, con uso frecuente del afrontamiento religioso-espiritual. Se analizó el proceso de afrontamiento del 20 madres (17-39 años), con bebés hospitalizados en la UCIN ( $M=10$ días). Se aplicó la Escala de Adaptación Religiosa-Espiritual - CRE ( 87 ítems), dispuestos en 8 factores de CREP (positivo) y 4 CREN (negativo). Analizamos sus relaciones con categorias adaptativas de afrontamiento (autoconfianza, búsqueda de apoyo, solución de problemas, búsqueda de información, alojamiento, negociación) y desadaptativas (Delegación, aislamiento, desamparo, la fuga, la sumisión y la oposición) propuestas por el TMC. Los índices de Escala CRE eran, en promedio, con un predominio de CREN y mayor frecuencia de posicionamiento negativo delante de Dios, y prevalencia de las estrategias desadaptativas como presentación, Escape y Oposición. Hubo correlaciones entre CREP y familias de adaptación; y entre CREN y familias desadaptativas. Fue posible no solo identificar el uso del afrontamiento religioso y espiritual en esta muestra, pero también analizar sus relaciones con proceso de adaptación, expandiendo las posibilidades de análisis de Escala CRE.

Palabras clave: Coping, estrés, Unidades de Cuidado Intensivo Neonatal, Escala de Afrontamiento Religioso-Espiritual.

Os conceitos de estresse e coping (enfrentamento) são interligados. O estresse é compreendido como uma reação do organismo, que inclui componentes físicos, psicológicos, mentais e hormonais, que são gerados pela necessidade de lidar com algum evento percebido enquanto ameaça. Considera-se que as reações de estresse fazem parte de um mecanismo adaptativo, me- 
diado pela capacidade de superação das adversidades, a qual depende da habilidade de lidar com as mudanças, ou seja, das estratégias de enfrentamento (EE) do indivíduo (Aldwin, 2011).

Uma das grandes fontes estressoras é o contexto hospitalar, tendo em vista que é um ambiente relacionado ao risco à vida, especialmente nas Unidades de Terapia Intensiva Neonatal (UTIN). Nesse ambiente, têm-se as condições de instabilidade orgânica do bebê, a necessidade de cuidados médicos especializados, a separação do bebê, a incerteza sobre a evolução clínica e sobrevivência, a distorção entre imagem do "bebê idealizado" e o "bebê real", e o ambiente estranho da UTIN. Todas estas situações configuram-se como estressores, de intensa ansiedade e imprevisibilidade para algumas famílias (Guimarães, Paula, \& Enumo, 2013; Pinheiro, Balbino, Balieiro, Domenico, \& Avena, 2009; Roseiro \& Paula, 2015), com consequências também para a saúde mental das mães (Fraga, Linhares, Carvalho, \& Martinez, 2008; Melnyk, Feinstein, \& Fairbanks, 2006; Ramos, 2012). Saber enfrentar essa situação se torna imprescindível nesse momento em que o bebê necessita de total atenção.

$\mathrm{Na}$ tentativa de administrar o estresse, a religião e a espiritualidade são recursos frequentemente acionados, constituindo-se o que se denomina como "enfrentamento religioso-espiritual" (spiritual-religious coping; Almeida \& Stroppa, 2010; Alves, Alves, Barboza, \& Souto, 2010; Pargament, 2011; Véras, Vieira, \& Morais, 2010). Lamy et al. (2011) afirmam que o uso do enfrentamento religioso-espiritual pode se dar pela crença em um Deus facilitador na recuperação do bebê, que dá forças para o enfrentamento mesmo diante dos desafios percebidos na UTIN. Segundo os autores, na fase inicial, caracterizada pelo recebimento da má notícia e do medo da morte, a fé é um consolo e uma esperança, enquanto que, na fase final da internação, delega-se a Deus a melhora e a alta hospitalar que se aproxima. Durante todo esse processo de internação do bebê, ou mesmo antes, na gestação de risco, assim como em outras condições de doenças, para lidar com essa condição estressante, o uso da religião e da espiritualidade é frequente (Benute et al., 2011; Gobatto \& Araújo, 2010).
Uma definição recente de coping explica o processo de enfrentamento com uma abordagem desenvolvimentista, centrada na autorregulação, pela Teoria Motivacional do Coping - TMC (Skinner, Edge, Altman, \& Sherwood, 2003; Skinner \& Wellborn, 1994; Zimmer-Gembeck \& Skinner, 2011). O coping é definido pela TMC como um constructo organizacional que descreve ". . c como as pessoas regulam seus próprios comportamentos, emoções e orientações motivacionais sobre condições estressantes" (Skinner \& Wellborn, 1994, p. 107).

A TMC considera que os estressores são eventos que ameaçam ou desafiam as necessidades básicas de relacionamento ou vinculação com outras pessoas, de competência e de autonomia (Ramos, Enumo, \& Paula, 2015; Skinner et al., 2003; Skinner \& Wellborn, 1994). O processo de enfrentamento inclui as consequências das EE utilizadas e os efeitos no curto e longo prazo, os quais podem ser analisados em termos de serem adaptativos ou mal adaptativos (Skinner \& Wellborn, 1994). Após ampla revisão da área, Skinner et al. (2003) propuseram, conforme a avaliação do estressor como ameaça ou desafio às necessidades básicas de autonomia, competência ou de relacionamento, 12 categorias de alta ordem, denominadas "famílias de coping". Estas são multidimensionais e multifuncionais e fazem ligação com os processos adaptativos. Quando o estressor é visto como um desafio, tem-se as famílias adaptativas: Autoconfiança, Busca de Suporte, Resolução de Problemas, Busca de Informações, Acomodação, Negociação; e se o estressor é visto como uma ameaça, têm-se as famílias de coping mal adaptativas no médio e longo prazo: Delegação, Isolamento, Desamparo, Fuga, Submissão e Oposição.

Para avaliar o CRE, tem sido usada em nosso país uma adaptação do Religious Coping Questionnaire - RCOPE (Pargament, Koenig, \& Peres, 2000) - a Escala de Coping Religioso-Espiritual - CRE (Panzini \& Bandeira, 2005), cujos itens foram organizados com base na análise fatorial. Considerando que os comportamentos avaliados na Escala CRE podem também ser analisados sob a TMC, procurou-se aqui identificar outras funções adaptativas, diferentes daque- 
las obtidas pela análise fatorial. Dessa forma, a análise do processo de enfrentamento poderia ir além dos dados descritivos obtidos com a aplicação tradicional da escala, buscando-se suas relações com as categorias de alta ordem propostas pela TMC. Assim, o objetivo do presente estudo foi analisar as estratégias de enfrentamento de mães com bebês internados em UTIN, com destaque para o enfrentamento religioso-espiritual e suas relações com processos adaptativos, segundo a TMC.

\section{Método}

\section{Participantes e Local de Coleta de Dados}

Participaram 20 mães de bebês internados em UTIN de uma cidade do interior do estado de São Paulo, com idade entre 17 e 39 anos $(M=$ $25,00 ; \pm 6,64)$, compondo uma amostra de conveniência. A maioria $(n=17)$ pertencia à classe socioeconômica $\mathrm{D}-\mathrm{E}$ e $\mathrm{C} 1$, que equivalem à renda familiar de $\mathrm{R} \$ 895,00$ e $\mathrm{R} \$ 1.865,00$, respectivamente ( $M=$ US\$520.00, considerando o dólar a 2,65 reais, em dezembro de 2014), segundo o Critério de Classificação Econômica Brasil, da Associação Brasileira de Empresas de Pesquisa (ABEP, 2013). A maioria das mães não completou o Ensino Médio $(n=20)$, era casada ou vivia em união estável $(n=15)$ e nunca teve filho prematuro $(n=13)$. Todas as mães afirmaram sua crença em Deus, sendo a maioria da religião católica $(n=15)$, mas não praticante da religião $(n=11)$.

Houve maioria de mães $(n=14)$ multíparas; mas, mesmo aquelas consideraram sua gravidez de risco $(n=6)$ demonstraram pouco conhecimento sobre o significado do alto risco gestacional. Dezesseis mães relataram não ter problemas de saúde; mas, quatro apresentaram quadros clínicos, como o Diabetes Gestacional $(n=2)$. Doze mães fizeram entre 5 e 7 consultas pré-natais.

A maioria dos partos dessas mães foi feito por cesariana $(n=11)$, nascendo mais bebês do sexo feminino $(n=14)$. A idade gestacional dos bebês variou de 35 a 38 semanas, com predomínio de nascimentos prematuros $(n=11)$, sendo
8 prematuros moderados (32-36 semanas), um muito prematuro (28-32 semanas) e 2 extremamente prematuros ( $<28$ semanas). Os nascimentos a termo, com mais de 37 semanas de gestação, somaram 9 bebês. O peso ao nascimento variou de $800 \mathrm{~g}$ a $3.780 \mathrm{~g}$, havendo 8 bebês com peso normal $(\geq 2.500 \mathrm{~g}$ ), próximo ao número de nascimentos de bebês com baixo peso ( $n=$ 7). Assim, os motivos para internação na UTIN centraram-se na prematuridade e/ou baixo peso, havendo também casos de infecção e problemas respiratórios.

O tempo de internação variou de 1-181 dias $(M=10,50 ; \pm 39,76)$, com maior incidência de internação por três dias $(n=3)$. Durante a internação, a maioria dos bebês teve alguma intercorrência $(n=18)$, como: infecção $(n=5)$, uma combinação de quadros clínicos $(n=5)$, problemas respiratórios $(n=4)$ ou cardíacos $(n=2)$, perda de peso $(n=1)$ e fenda palatina $(n=1)$.

\section{Instrumento}

Escala de Coping Religioso-Espiritual Escala CRE (Panzini, 2004; Panzini \& Bandeira, 2005) - é uma adaptação do Religious Coping Questionnaire (RCOPE Scale; Pargament et al., 2000) para avaliação do enfrentamento religioso-espiritual em adultos. Esta escala apresenta indicadores de utilização positiva ou negativa do enfrentamento religioso-espiritual, em situações de estresse. A escala original (RCOPE) tem 21 subescalas, cada qual contendo 5 itens, respondidos em escala de 4 pontos (0- "not at all"; 3- "a great deal"); apresenta um Alpha de Cronbach maior que 0,80 em cada subescala.

Após validação linguística e adaptação da RCOPE, a aplicação em 616 brasileiros e a análise fatorial dos dados geraram a Escala CRE (Panzini \& Bandeira, 2005). Esta tem 87 itens, em escala tipo Likert de cinco pontos, variando entre "nem um pouco" e "muitíssimo". Os itens são divididos em duas dimensões:

1. Dimensão CRE Positivo (CREP; oito fatores): (a) Transformação de si e/ou sua vida; (b) Ações em busca de ajuda espiritual; (c) Oferta de ajuda ao outro; (d) Posicionamento positivo frente a Deus; (e) Busca pessoal de crescimento pessoal; (f) Ações em busca 
do outro institucional; (g) Busca pessoal de conhecimento espiritual; (h) Afastamento através de Deus, da religiosidade e/ou espiritualidade, totalizando 66 itens); e,

2. Dimensão CRE Negativo (CREN; quatro fatores): (a) Reavaliação negativa de Deus; (b) Posicionamento negativo frente a Deus; (c) Reavaliação negativa do significado; (d) Insatisfação com o outro institucional, totalizando 21 itens). A Escala CRE apresenta confiabilidade ótima pelo Alpha de Cronbach $(\alpha \geq 0,70)$, para o conjunto de itens $(\alpha=$ $0,97)$, para a Dimensão $\operatorname{CREP}(\alpha=0,98)$ e para a Dimensão CREN ( $\alpha=0,86$; Panzini \& Bandeira, 2005).

Critério de Classificação Econômica Brasil - CCEB (ABEP, 2013) - é uma escala de dados socioeconômicos que, a partir do estabelecimento de cinco classes econômicas, sendo as três primeiras subdivididas para possibilitar uma distribuição mais consistente com a realidade brasileira (A1, A2, B1, B2, C1, C2, D e E), permitiu caracterizar o nível socioeconômico da amostra.

\section{Procedimento}

Após aprovação do estudo pelo Comitê de Ética em Pesquisa da universidade, pelo Parecer $\mathrm{n}^{\mathrm{o}} 536.014$, os dados foram coletados no próprio hospital em que os bebês estavam internados, em uma cidade do interior do estado de São Paulo, no período de 28 de abril a 8 de setembro de 2014 (4 meses e 10 dias). O contato com as mães só era possível nos horários de visita da UTIN (13-14 h ou das 19-20 h) e esta sempre acontecia ao final de cada visita na porta da UTIN.

A coleta foi realizada em 21 sessões, com uma duração média de 55 minutos. Aquelas que aceitavam participar da entrevista eram dirigidas a uma sala disponibilizada pela direção da instituição localizada no corredor do Centro de Terapia Intensiva. Nesta ocasião, era apresentado o Termo de Consentimento Livre e Esclarecido (TCLE) e entregue os instrumentos, explicando anteriormente como deveriam ser respondidos. Algumas entrevistadas preferiam falar enquanto a pesquisadora ou as auxiliares anotavam as informações que eram prestadas. Ao término de cada entrevista, a pesquisadora ou auxiliares agradeciam a participação e anotavam o Escore Apgar do bebê, com a equipe de enfermagem.

\section{Análise de Dados}

A Escala CRE teve seus escores calculados com base nas médias, como indicado por Panzini e Bandeira (2005). Primeiro, calcula-se a média para cada fator do instrumento, em seguida, faz-se a média dos oito fatores correspondentes ao CREP (positivo), com 66 itens (P1+P2+P3+P4+P5+P6+P7+P8/8). Faz-se o mesmo para os quatro fatores do CREN (negativo), com 21 itens $(\mathrm{N} 1+\mathrm{N} 2+\mathrm{N} 3+\mathrm{N} 4 / 4)$. O CRE Total é a média entre o Índice CREP (positivo) e a média das respostas invertidas aos 21 itens CREN (CRE Negativo Invertido). Essa média pode variar de 1 a 5, sendo classificada em Irrisória ou Nenhuma (1 a 1,5), Baixa (1,51 a 2,5), Média (2,51 a 3,5), Alta (3,51 a 4,5) e Altíssima (4,51 a 5) (Panzini, 2004). Assim, quanto mais alto o escore, maior é a utilização de CRE. Por fim, é calculada a razão CREN/CREP pela divisão simples entre os índices básicos, em relação inversamente proporcional: quanto mais baixo resultar seu valor, maior é o uso de CREP em relação ao de CREN, e vice-versa (Panzini \& Bandeira, 2005).

Categorização da Escala CRE pela Teoria Motivaional do Coping e Concordância Entre Avaliadores. Para a classificação da Escala CRE pela TMC (Skinner et al., 2003), foram categorizados pela pesquisadora os 87 itens segundo as 12 famílias de coping. Essa categorização inicial foi avaliada por três juízes (um pesquisador, um doutorando e um mestrando, todos com formação em Psicologia), selecionados por pertencerem ao grupo de pesquisa, realizando estudos e pesquisa sobre o enfrentamento do estresse segundo a TMC. Os juízes receberam por e-mail a Classificação das Estratégias de Enfrentamento nas Famílias de Enfrentamento, contendo: informações sobre as famílias do enfrentamento do estresse segundo a TMC, um breve embasamento teórico, seguido de uma tabela contendo a lista de questões da Escala CRE e as famílias de coping, para que pudessem ser assinaladas, 
indicando qual família cada item da Escala CRE pertencia.

A concordância entre os juízes variou de $64,5 \%$ a $77 \%$, obtendo-se uma concordância geral de 76,16. Esta pode ser considerada adequada para este tipo de comparação que envolve muitas categorias complexas $\%$ ( $>70 \%$, segundo Fagundes, 2006). Essa nova classificação da Es- cala CRE segundo a TMC mostrou que a Escala CRE tem mais EE adaptativas $(67,84 \%)$ do que mal adaptativas (32,20\%). Das 12 famílias do enfrentamento segundo a TMC, a mais frequente na Escala CRE é a Busca de Suporte (25,29\%), seguida por Acomodação $(12,64 \%)$. Entre as famílias mal adaptativas de coping, as mais frequentes nessa escala são Delegação $(8,05 \%)$ e Oposição $(6,90 \%$; Tabela 1).

\section{Tabela 1}

Itens da Escala de Coping Religioso-Espiritual (CRE) segundo as Categorias de Enfrentamento da Teoria Motivacional do Coping

\begin{tabular}{|c|c|c|c|}
\hline $\begin{array}{l}\text { Famílias do } \\
\text { Enfrentamento }\end{array}$ & Descrição* & Itens da Escala CRE & Total $(\%)$ \\
\hline Autoconfiança & $\begin{array}{l}\text { Tentativa de regulação emocional, } \\
\text { comportamental, expressão emocional } \\
\text { e aproximação emocional. }\end{array}$ & $25,34,43,47,49,55,63,85$ & $8(9,19)$ \\
\hline Busca de suporte & $\begin{array}{l}\text { Busca de contato, conforto, ajuda } \\
\text { instrumental, referenciamento social. }\end{array}$ & $\begin{array}{l}2,5,8,11,12,13,14,17 \\
19,21,26,29,44,48,57 \\
58,60,66,74,80,81,87\end{array}$ & $22(25,29)$ \\
\hline $\begin{array}{l}\text { Resolução de } \\
\text { problemas }\end{array}$ & $\begin{array}{l}\text { Planejar estratégias, ações instrumentais, } \\
\text { planejamento, domínio. }\end{array}$ & $\begin{array}{c}28,30,39,40,45,67 \\
68,71,75\end{array}$ & $9(10,34)$ \\
\hline Busca de informações & Ler, observar, perguntar a outros. & $16,38,52,54,72,77$ & $6(6,90)$ \\
\hline Acomodação & $\begin{array}{l}\text { Distração e/ou reestruturação cognitiva, } \\
\text { minimização, aceitação. }\end{array}$ & $\begin{array}{c}1,3,10,24,31,33,62 \\
65,70,79,86\end{array}$ & $11(12,64)$ \\
\hline Negociação & $\begin{array}{l}\text { Barganha, persuasão, } \\
\text { estabelecimento de prioridades. }\end{array}$ & $9,46,56$ & $3(3,48)$ \\
\hline Total adaptativas & & & $59(67,84)$ \\
\hline Delegação & $\begin{array}{l}\text { Reclamação, autoculpa, lamentação, } \\
\text { busca de suporte mal adaptativo. }\end{array}$ & $7,22,23,35,59,61,64$ & $7(8,05)$ \\
\hline Isolamento & $\begin{array}{l}\text { Afastamento, evitação social dissimulação, } \\
\text { paralisar. }\end{array}$ & $18,36,69,73,76$ & $5(5,75)$ \\
\hline Desamparo & $\begin{array}{l}\text { Confusão, interferência, exaustão cognitiva, } \\
\text { passividade. }\end{array}$ & $15,32,41,50,51$ & $5(5,75)$ \\
\hline Fuga & $\begin{array}{c}\text { Afastamento mental, negação, pensamento } \\
\text { desejoso. }\end{array}$ & $20,27,42,82$ & $4(4,60)$ \\
\hline Submissão & $\begin{array}{l}\text { Ruminação, pensamentos intrusivos, } \\
\text { perseveração rígida. }\end{array}$ & 37 & $1(1,15)$ \\
\hline Oposição & Culpar outros, projeção, agressão, desafiar. & $4,9,53,78,83,84$ & $6(6,90)$ \\
\hline Total mal adaptativas & & & $28(32,20)$ \\
\hline
\end{tabular}

Nota. *Adaptado de Ramos (2012, p. 63, em tradução livre para pesquisa baseada em Skinner \& Zimmer-Gembeck, 2007, 2009; Skinner et al., 2003; Zimmer-Gembeck \& Skinner, 2008), e de Pargament et al. (2000); CRE = Escala de Coping Religioso-Espiritual (Panzini \& Bandeira, 2005). 
Após essa nova classificação da Escala CRE, foram identificadas as famílias de coping mais utilizadas pelas mães da amostra, calculando-se a média de cada participante para cada família de coping. Em seguida, utilizou-se o teste estatístico não paramétrico de correlação de Spearman, para verificar a concordância entre essas classificações propostas, considerando um nível de significância de $p \leq 0,05$. Para a análise das relações de influência entre as duas classificações (se a função do enfrentamento religioso-espiritual proposta pela Escala CRE influenciava significativamente as famílias de coping), foi utilizado o teste não paramétrico de Kruskal-Wallis, considerando um nível de significância de $p \leq 0,05$. Para as análises estatísticas, utilizou-se o pacote estatístico SPSS® ${ }^{18.0 .}$

Tabela 2

Enfrentamento Materno da Internação do Bebê na UTIN, pela Escala de Coping Religioso-Espiritual (CRE; $N=20)$

\begin{tabular}{|c|c|c|c|c|c|}
\hline Fatores da Escala CRE & $\begin{array}{l}\mathrm{N}^{\mathrm{o}} \mathrm{de} \\
\text { itens }\end{array}$ & $f(\%)$ & $\begin{array}{l}\text { Valor } \\
\text { Mínimo }\end{array}$ & $\begin{array}{l}\text { Valor } \\
\text { Máximo }\end{array}$ & $\begin{array}{c}\text { Média } \\
\text { (Desvio-padrão) }\end{array}$ \\
\hline Índice CRE Total* & 87 & 100,00 & 1,15 & 3,83 & $3,08( \pm 0,62)$ \\
\hline Índice CREP (positivo) & 66 & 75,85 & 1,74 & 3,92 & $2,93( \pm 0,67)$ \\
\hline Índice CREN (negativo) & 21 & 24,15 & 1,09 & 4,09 & $2,14( \pm 0,83)$ \\
\hline Razão CREN/CREP** & & & 0,45 & 2,83 & $0,83( \pm 0,51)$ \\
\hline \multicolumn{6}{|l|}{ CREP } \\
\hline P1 - Transformação de si e/ou de sua vida & 14 & 16,09 & 1,00 & 4,71 & $3,34( \pm 1,02)$ \\
\hline P2 - Ações em busca de ajuda espiritual & 8 & 9,20 & 1,12 & 3,87 & $2,17( \pm 0,73)$ \\
\hline P3 - Oferta de ajuda ao outro & 7 & 8,05 & 1,57 & 3,71 & $2,53( \pm 0,85)$ \\
\hline P4 - Posicionamento positivo frente a Deus & 11 & 12,64 & 3,81 & 4,72 & $4,25( \pm 0,36)$ \\
\hline P5 - Busca pessoal de crescimento espiritual & 5 & 5,75 & 1,40 & 4,20 & $2,76( \pm 0,85)$ \\
\hline P6 - Ações em busca do outro institucional & 10 & 11,49 & 1,10 & 3,90 & $2,19( \pm 0,79)$ \\
\hline P7 - Busca pessoal de conhecimento espiritual & 5 & 5,75 & 1,00 & 3,80 & $1,88( \pm 0,93)$ \\
\hline $\begin{array}{l}\text { P8 - Afastamento através de Deus/religião/ } \\
\text { espiritualidade }\end{array}$ & 6 & 6,90 & 1,83 & 4,16 & $3,25( \pm 0,81)$ \\
\hline \multicolumn{6}{|l|}{ CREN } \\
\hline N1 - Reavaliação negativa de Deus & 8 & 9,20 & 1,00 & 3,25 & $1,82( \pm 0,78)$ \\
\hline N2 - Posicionamento negativo frente a Deus & 4 & 4,60 & 1,25 & 4,75 & $2,83( \pm 1,18)$ \\
\hline N3 - Reavaliação negativa do significado & 5 & 5,75 & 1,00 & 3,40 & $2,34( \pm 0,85)$ \\
\hline N4 - Insatisfação com o outro institucional & 4 & 4,60 & 1,00 & 2,25 & $1,73( \pm 0,86)$ \\
\hline
\end{tabular}

Nota. *variação =1,00 a 5,00; **variação = 0,20 a 5,00; quanto menor a razão CREN/CREP, melhor o resultado, com CREP maior; UTIN = Unidade de Terapia Intensiva Neonatal.

\section{Resultados}

As mães apresentaram todos os índices de CRE na "média", inclusive para o CRE Total $(\mathrm{CRET}=3,08)$, representando a quantidade total de CRE praticado pela amostra. Na Dimensão CREP (coping positivo; CREP $=2,93$ ), o Fator P4 - Ter um posicionamento positivo frente a Deus - foi o mais utilizado (Tabela 2), representando uma maior conexão com Deus, reavaliação positiva do estressor através Dele, contemplando comportamentos como contar, colaborar, independentemente da ajuda de Deus. A Dimensão CREN (coping negativo) também apresentou um índice na "média" (CREN = 2,14). O Fator N2 Posicionamento Negativo frente a Deus -foi mais utilizado (Tabela 2), representando delegação religiosa, passiva ou súplica negativa, atribuindo a Deus o controle para a resolução do problema. 
A razão de CREN/CREP refere-se ao uso de EE religioso-espiritual negativas em relação às positivas. Nesta amostra, a razão obtida indica que as mães usaram $73 \%$ de coping religioso-espiritual negativo em relação ao coping religioso-espiritual positivo, demonstrando a prevalência de estratégias negativas de enfrentamento religioso-espiritual (Tabela 2).

Ao analisar as famílias do enfrentamento pela TMC para as respostas das mães à Escala CRE, foi encontrada maior frequência de $S u b$ missão $(n=6)$, seguido de Fuga $(n=4)$, Oposição $(n=2)$, Busca de Suporte $(n=2)$. As demais famílias de enfrentamento tiveram uma frequência mínima $(n=1)$, o que demonstra que a maior frequência de EE foram mal adaptativas.

Através do teste estatístico de KruskallWallis, não foram encontradas influências significativas entre as variáveis independentes (famílias de coping) e a variável dependente
(CRE Total), pois o nível de significância foi maior que 0,05 . Contudo, foram encontradas correlações significativas e positivas entre Autoconfiança e P1 (Transformação de si e/ou sua vida); Acomodação e P3 (Oferta de ajuda ao outro); Busca de Suporte e N2 (Posicionamento negativo frente a Deus), P5 (Busca pessoal de crescimento pessoal), P6 (Ações em busca do outro institucional) e P7 (Busca pessoal de conhecimento espiritual). Também houve correlações negativas entre Delegação e N2 (Posicionamento negativo frente a Deus), indicando que, quanto menor é a utilização de EE com a função de delegar ao outro as responsabilidades, maior será o posicionamento negativo frente a Deus, e vice-versa; Fuga e P5 (Busca pessoal de crescimento pessoal), indicando que, quanto mais a pessoa utiliza EE com a função de Fuga, menos ela busca crescimento pessoal e vice-versa (Tabela 3 ).

Tabela 3

Correlação entre Fatores da Escala CRE e as Famílias de Coping da Teoria Motivacional do Coping

\begin{tabular}{|c|c|c|c|c|c|c|c|c|c|c|c|c|}
\hline $\begin{array}{l}\text { TMC } \\
\text { CRE }\end{array}$ & 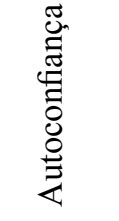 & 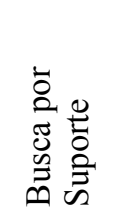 & 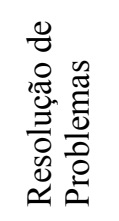 & 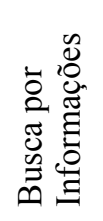 & 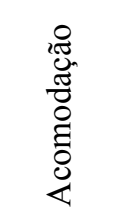 & 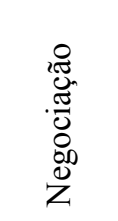 & 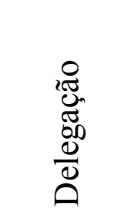 & 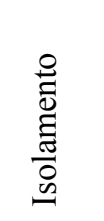 & 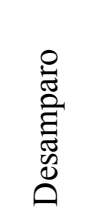 & 总 & 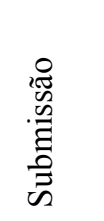 & 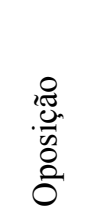 \\
\hline $\mathrm{N} 1$ & 0,22 & 0,07 & 0,10 & 0,22 & 0,03 & 0,06 & $-0,27$ & 0,14 & $-0,16$ & $-0,13$ & 0,02 & $-0,12$ \\
\hline $\mathrm{N} 2$ & 0,37 & $0,60 * *$ & 0,41 & 0,39 & 0,41 & 0,31 & $-0,59^{* *}$ & $-0,21$ & $-0,11$ & $-0,28$ & $-0,19$ & $-0,20$ \\
\hline N3 & 0,42 & 0,31 & 0,27 & 0,33 & 0,22 & 0,13 & $-0,30$ & $-0,26$ & $-0,12$ & $-0,24$ & $-0,07$ & $-0,26$ \\
\hline N4 & 0,13 & 0,01 & $-0,07$ & 0,13 & 0,00 & $-0,24$ & $-0,22$ & 0,21 & $-0,08$ & $-0,65$ & 0,05 & 0,06 \\
\hline P1 & $0,48^{*}$ & 0,26 & 0,39 & 0,25 & 0,40 & 0,22 & $-0,06$ & 0,11 & 0,07 & $-0,28$ & $-0,08$ & 0,01 \\
\hline P2 & 0,21 & $-0,01$ & 0,14 & 0,26 & 0,12 & 0,13 & $-0,07$ & 0,19 & 0,06 & $-0,34$ & 0,13 & $-0,02$ \\
\hline P3 & 0,30 & 0,41 & 0,43 & 0,35 & $0,48^{*}$ & 0,30 & $-0,17$ & $-0,11$ & $-0,07$ & $-0,43$ & $-0,06$ & $-0,01$ \\
\hline P4 & $-0,22$ & 0,42 & $-0,07$ & $-0,20$ & 0,11 & $-0,03$ & 0,08 & 0,08 & 0,16 & $-0,13$ & 0,36 & 0,21 \\
\hline P5 & 0,22 & $0,49 *$ & 0,34 & 0,26 & 0,31 & 0,27 & $-0,37$ & $-0,02$ & $-0,15$ & $-0,51 *$ & 0,08 & $-0,17$ \\
\hline P6 & 0,29 & $0,55^{*}$ & 0,30 & 0,16 & 0,40 & 0,12 & $-0,16$ & 0,15 & 0,10 & $-0,93$ & $-0,05$ & 0,02 \\
\hline P7 & 0,38 & $0,49^{*}$ & 0,32 & 0,34 & 0,36 & 0,20 & $-0,28$ & $-0,31$ & $-0,03$ & $-0,35$ & $-0,30$ & $-0,18$ \\
\hline P8 & 0,31 & 0,41 & 0,30 & 0,21 & 0,28 & 0,14 & 0,00 & $-0,04$ & 0,22 & $-0,42$ & $-0,10$ & 0,07 \\
\hline $\begin{array}{l}\text { Razão } \\
\text { CRE }\end{array}$ & $-0,22$ & $-0,34$ & $-0,24$ & 0,16 & $-0,26$ & $-0,34$ & 0,28 & 0,36 & 0,28 & 0,14 & 0,00 & 0,32 \\
\hline
\end{tabular}

Nota. CRE = Escala de Coping Religioso-Espiritual (Panzini \& Bandeira, 2005); $\mathrm{N}=$ negativo; $\mathrm{P}=$ positivo. ${ }^{*} p \leq 0,05 ; * * \leq 0,01$, significativo pelo teste de Kruskal-Wallis. 


\section{Discussão}

Entre as diversas formas de lidar com o estresse, a religião e a espiritualidade são formas comuns de enfrentamento. Mesmo considerando a religião uma fonte acessível a todos de suporte social e instrumental para a resolução de problemas (Gobatto \& Araujo, 2010; Pargament, 2011), deve ser feita uma cuidadosa análise quanto à sua funcionalidade no enfrentamento do estresse.

Nesse sentido, é relevante analisar o enfrentamento em suas mais variadas formas de comportamento, compreendendo-o como um processo, com seus eventos antecedentes e suas consequências. Analisa-se, assim, a funcionalidade do comportamento de enfrentamento em relação às condições disponíveis para o indivíduo enfrentar o estressor e as consequências de curto e longo prazo, as quais podem ser adaptativas ou mal adaptativas (Ramos et al., 2015; Skinner \& Wellborn, 1994).

Estudar o coping religioso-espiritual em mães frente à internação do filho em UTIN usando a Escala CRE (Panzini \& Bandeira, 2005), com dados quantitativos, traz dados interessantes. A razão CREN/CREP (EE negativas/positivas), neste estudo, demonstrou uma prevalência de estratégias religiosas/espirituais negativas para lidar com a internação do filho em UTIN. Estes dados enriquecem os resultados já apontados por outras pesquisas, que indicam qualitativamente o uso frequente do coping religioso-espiritual no contexto da UTIN (Lamy et al., 2011; Ramos, 2012; Véras et al., 2010) e da hospitalização (Gobatto \& Araújo, 2010; Taunay et al., 2012). Lidar com a internação do filho em UTIN implica em administrar um ambiente novo e imprevisível, o que tende a ser feito por meio de EE pouco adaptativas (Fraga et al., 2008; Ramos, 2012). Confirmando, neste estudo, foi encontrada uma frequência maior de EE mal adaptativas, indicando uma percepção dos estressores como uma ameaça, tanto que as famílias de coping mais utilizadas foram Submissão, Fuga e Oposição.

Analisando a primeira família de coping mais frequente, Submissão, entende-se que sub- meter-se a essa condição da UTIN pode resultar em uma percepção de impotência para resolver os problemas dessa situação, implicando em ter pensamentos intrusivos, repetitivos e centrados em aspectos negativos (ruminação), ou seja, um posicionamento passivo diante da situação estressante. A Fuga, por sua vez, também traz características de passividade e percepção negativa sobre os recursos próprios para lidar com o problema. Opor-se, culpando outros, também se refere a uma forma passiva de enfrentamento, que pode implicar em um relacionamento disfuncional com o bebê, não promotor do desenvolvimento deste e da saúde dessas mães. Essas consequências, portanto, são prejudiciais à mãe, ao bebê e às futuras gestações dessas mães (Almeida \& Stroppa, 2010; Alves et al., 2010; Pargament, 2011). Isto decorre do fato do coping religioso-espiritual negativo estar relacionado a piores resultados de saúde, uso impróprio de serviços de saúde, pior qualidade de vida, depressão, insensibilidade para com os outros, maior nível de estresse, culpa e medo, por exemplo (Almeida \& Stroppa, 2010; Alves et al., 2010; Benute et al., 2011; Pargament, 2011).

Em contrapartida, quando utilizadas de modo adaptativo, a religiosidade e a espiritualidade implicam em melhores resultados de saúde, sendo a religião uma instituição provedora de suporte social, capaz de atribuir significados aos reveses da vida e instrumentalizar para resolução de problemas (Gobatto \& Araujo, 2010; Goulart, Somarriba, \& Xavier, 2005; Lamy et al., 2011; Ramos, 2012). Neste estudo, os resultados referentes ao uso adaptativo da religião e da espiritualidade e a família de coping mais utilizada indicam o reconhecimento de Deus como colaborador para enfrentar a desafiante situação de internação do filho em UTIN.

As correlações convergem com o uso comum do enfrentamento religioso-espiritual, uma vez que a religião e a fé permitem significação, ordenação da vida, de seus revezes e sofrimento (Pargament, 2011), incluindo momentos de revolta e atribuição de culpa aos céus e seus desígnios. A religião, quando utilizada como forma de enfrentamento positiva (CREP), inclui EE relacionadas à Autoconfiança e à Busca de Suporte, 
indicando que as mães se sentiram desafiadas em sua necessidade de Relacionamento, agindo de forma adaptativa. Buscam, com isso, transformar sua vida, a si mesma, e crescer pessoal e espiritualmente, com uma orientação motivacional direcionada ao self (Skinner et al., 2003). Em contrapartida, houve uso da família de coping de Acomodação, na qual o estressor é percebido como um desafio à necessidade de Autonomia, indicando a condição de aceitação da internação do filho e a percepção de sua necessidade. Esta família de coping apresentou correlação com a Oferta de Ajuda ao Outro, o que faz sentido, uma vez que a adaptação à situação implica em apresentar comportamentos de cooperação com o outro.

No entanto, o coping religioso-espiritual mal adaptativo (CREN) implica em uma relação oposta com Deus, ou sentindo-se abandonado por Ele, com busca de suporte em outras pessoas, inclusive agentes religiosos, estratégias estas que apresentaram também correlação com Ações em Busca do Outro Institucional. Diante desse panorama, entende-se como condição preocupante o uso restrito de apoio em agentes religiosos, por não viabilizar a inclusão da mãe no cuidado com os bebês. Essa participação das mães traz benefícios, como o aumento da competência dos pais no cuidado com o bebê após a alta, a redução da tensão vivida no período da internação e o aumento da prevalência do aleitamento materno (Guimarães et al., 2013; Roseiro \& Paula, 2015).

Em termos motivacionais, quanto menos a necessidade de Relacionamento é ameaçada, ou seja, quanto menos a mãe entende que encontrou limites em seus recursos sociais disponíveis, menor é o uso de EE de Delegação. Delegar, depender e/ou exigir de outros a solução de problemas, com sentimentos de autopiedade, lamentação e vergonha, por sua vez, aumenta o posicionamento negativo frente a Deus, com as consequências de médio e longo prazo discutidas.

\section{Considerações Finais}

Compreender de maneira detalhada e abrangente o processo de enfrentamento do estresse pode facilitar no direcionamento de propostas de intervenções a essa população de mães de prematuros internados em UTIN, uma vez que a descrição e análise das estratégias de enfrentamento utilizadas ficam mais claras e podem ser associadas aos processos adaptativos. Esta condição permite minimizar problemas de médio e longo prazo à mãe e ao bebê, uma vez que pode incentivar a reflexão acerca da funcionalidade da religião e da espiritualidade no processo de enfrentamento e o repertório comportamental adaptativo para lidar com o estresse.

Uma característica comum nas instituições de saúde refere-se à presença de religiosos no cuidado e apoio ao enfermo e sua família, condição esta que pode ser determinante na percepção do estresse, no enfrentamento e na análise dos resultados deste. Assim, compreender o enfrentamento do estresse a partir de uma análise mais ampla, que contemple também as estratégias religiosas/espirituais, permite subsidiar programas de intervenção mais efetivos.

Mediante essas condições, é relevante a realização de intervenções voltadas à promoção de estratégias de enfrentamento adaptativas. Por exemplo, pode-se tornar a Busca de Suporte mais funcional, com ações instrumentais por parte do setor de Serviço Social do hospital, orientações sobre outros comportamentos que podem diminuir o nível de estresse da mãe ou da família, como fazer perguntas à equipe e pedidos assertivos de ajuda, entre outros.

Para facilitar o enfrentamento adaptativo, é importante não apenas dar atenção às mães e familiares por parte dos profissionais, mas também planejar o acesso do suporte religioso-espiritual comum em muitas instituições de saúde. Este suporte não deve ser topograficamente definido, mas funcionalmente analisado, uma vez que as crenças religiosas e espirituais podem interferir na avaliação do estressor e nas estratégias de enfrentamento a serem utilizadas, com consequências para a saúde da mãe e do bebê.

Relacionar os resultados da Escala CRE com as famílias de enfrentamento propostas pela Teoria Motivacional do Coping contribuiu para o avanço teórico e metodológico da área do coping em nosso meio, trazendo reflexões sobre o 
processo de enfrentamento religioso-espiritual em conjunto mais amplo de recursos de enfrentamento. Esta condição permitiu compreender os outros recursos de enfrentamento utilizados na condição de internação do bebê em UTIN, fundamentais no processo de enfrentamento do estresse por essas mães. Pode também contribuir para estudos sobre a construção de novos instrumentos para avaliação nessa área, fornecendo um maior domínio de conhecimento sobre os recursos religiosos e espirituais para o enfrentamento do estresse.

\section{Referências}

Aldwin, C. (2011). Stress and coping across the lifespan. In S. Folkman (Ed.), The Oxford handbook of stress, health, and coping (pp. 15-84). New York: Oxford University Press.

Almeida, A. M., \& Stroppa, A. (2010). Espiritualidade \& Saúde Mental: Importância e impacto da espiritualidade na saúde mental. Zen Review, 2, 2-6.

Alves, R. R. N., Alves, H. N., Barboza, R. R. D., \& Souto, W. M S. (2010). The influence of religiosity on health. Ciência \& Saúde Coletiva, 15(4), 2105-2111. doi:10.1590/S141381232010000400024

Associação Brasileira de Empresas de Pesquisa. (2013). Critérios de Classificação Econômica Brasil (ABEP). Recuperado em http://www. abep.org/

Benute, G. R. G., Nomura, R. M. Y., Jorge, V. M. F., Nonnenmacher, D., Fráguas, R., Jr., Lucia, M. C. S., \& Zugaib, M. (2011). Risco de suicídio em gestantes de alto risco: Um estudo exploratório. Revista da Associação Médica Brasileira, 57(5), 583-587. doi:10.1590/S010442302011000500019

Fagundes, A. J. F. M. (2006). Descrição, definição e registro do comportamento (14. ed. rev. amp.). São Paulo, SP: Edicon.

Fraga, D. A., Linhares, M. B. M., Carvalho, A. E., V., \& Martinez, F. E. (2008). Desenvolvimento de bebês nascidos pré-termo e indicadores emocionais maternos. Psicologia: Reflexão e Crítica, 21(1), 33-41.

Gobatto, C. A., \& Araujo, T. C. C. F. (2010). Coping religioso-espiritual: Reflexões e perspectivas para a atuação do psicólogo em oncologia. Re- vista Sociedade Brasileira de Psicologia Hospitalar, 13(1), 52-63.

Goulart, L. M. H. F., Somarriba, M. G., \& Xavier, C. C. (2005). A perspectiva das mães sobre o óbito infantil: Uma investigação além dos números. Cadernos de Saúde Pública, 21(3), 715-723. doi:10.1590/S0102-311X2005000300005

Guimarães, F. H. C., Paula, K. M. P., \& Enumo, S. R. F. (2013). Estratégias de enfrentamento da prematuridade e suas relações com a amamentação no Alojamento Canguru. In P. R. M. Menandro \& R. S. Tokumaru (Eds.), Saúde, trabalho e familia: Multidisciplinaridade em foco (pp. 6-25). Vitória, ES: Programa de Pós-Graduação em Psicologia da Universidade Federal do Espírito Santo.

Lamy, Z. C., Morsch, D. S., Deslandes, S. F., Fernandes, R. T., Rocha, L. J. L. F., \& Lamy, F., Filho. (2011). Construção do papel materno a partir da vivência de internação em UTI Neonatal em dois modelos assistenciais. Revista Pesquisa Saúde, 12(1), 14-21.

Melnyk, B. M., Feinstein, N., \& Fairbanks, E. (2006). Two decades of evidence to support implementation of the COPE Program as standard practice with parents of young unexpectedly hospital$\mathrm{ized} /$ critically Ill children and premature infants. Pediatric Nursing, 32(5), 475-482.

Panzini, R. G. (2004). Escala de Coping Religioso-Espiritual (Escala CRE): Tradução, adaptação e validação da Escala RCOPE, abordando relações com saúde e qualidade de vida. Dissertação de mestrado, Universidade Federal do Rio Grande do Sul, Porto Alegre, RS, Brasil.

Panzini, R. G., \& Bandeira, D. R. (2005). Escala de Coping Religioso-Espiritual (Escala CRE): Elaboração e validação de construto. Psicologia em Estudo, 10(3), 507-516.

Pargament, K. I. (2011). Religion and coping: The current state of knowledge. In S. Folkman (Ed.), Oxford Handbook of stress, health, and coping (pp. 269-288). New York: Oxford University Press.

Pargament, K. I., Koenig, H. G., \& Peres, L. M. (2000). The many methods of religious coping: Development and initial validation of the RCOPE. Journal of Clinical Psychology, 56(4), 519-5423. doi:10.1002/ (SICI) 1097-4679(200004)56:4<519::AIDJCLP6>3.0.CO;2-1 
Pinheiro, E. M., Balbino, F. S., Balieiro, M. M. F. G., Domenico, E. B. L., \& Avena, M. J. (2009). Percepções da família do recém-nascido hospitalizado sobre a comunicação de más notícias. Revista Gaúcha de Enfermagem, 30(1), 77-84.

Ramos, F. P. (2012). Uma proposta de análise do coping no contexto de grupo de mães de bebês prematuros e com baixo peso na Unidade de Terapia Intensiva Neonatal. Tese de doutorado, Universidade Federal do Espírito Santo, Vitória, ES, Brasil).

Ramos, F. P., Enumo, S. R. F., \& Paula, K. M. P. (2015). Teoria Motivacional do Coping: Uma proposta desenvolvimentista de análise do enfrentamento do estresse. Estudos de Psicologia (Campinas), 32(2), 269-279. doi:10.1590/0103$-166 \mathrm{X} 2015000200011$

Roseiro, C. P., \& Paula, K. M. P. (2015). Concepções de humanização de profissionais em Unidades de Terapia Intensiva Neonatal. Estudos de Psicologia (Campinas), 32(1), 109-119. doi:10.1590/0103-166X2015000100010

Skinner, E. A., Edge, K., Altman, J., \& Sherwood, H. (2003). Searching for the structure of coping: A review and critique of category systems for classifying ways of coping. Psychological Bulletin, 129(2), 216-269. doi:10.1037/00332909.129.2.216
Skinner, E. A., \& Wellborn, J. G. (1994). Coping during childhood and adolescence: A motivational perspective. In D. L. Featherman, R. M. Lerner, \& M. Perlmutter (Eds.), Lifespan development and behavior (Vol. 12, pp. 91132). Hillsdale, NJ: Erlbaum.

Taunay, T. C. D., Gondim, F. A. A., Macêdo, D. S., Moreira-Almeida, A., Gurgel, L. A., Andrade, L. M. S., \& Carvalho, A. F. (2012). Validação da versão brasileira da escala de religiosidade de Duke (DUREL). Archives of Clinical Psychiatry, 39(4), 130-135. doi:10.1590/S010160832012000400003

Véras, R. M., Vieira, J. M. F., \& Morais, F. R. R. (2010). A maternidade prematura: O suporte emocional através da fé e religiosidade. Psicologia em Estudo, 15(2), 325-332.

Zimmer-Gembeck, M. J., \& Skinner, E. A. (2011). The development of coping across childhood and adolescence: An integrative review and critique of research. International Journal of Behavioral Development, 35(1), 1-17. doi: $10.1177 / 0165025410384923$

Recebido: 25/03/2015

$1^{a}$ revisão: 09/09/2015 Aceite final: 05/10/2015 